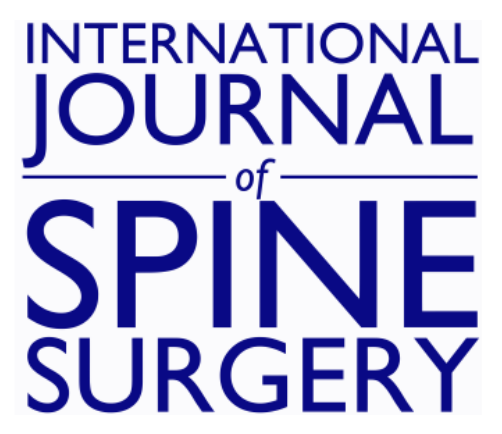

\title{
Endoscopic Spine Surgery of the Cervicothoracic Spine: A Review of Current Applications
}

Jian Shen, Elias Shaaya, Junseok Bae and Albert E. Telfeian

Int J Spine Surg 2021, 15 (suppl 3) S93-S103

doi: https://doi.org/10.14444/8168

http://ijssurgery.com/content/15/suppl_3/S93

This information is current as of April 26, 2023.

Email Alerts Receive free email-alerts when new articles cite this article. Sign up at:

http://ijssurgery.com/alerts 


\title{
Endoscopic Spine Surgery of the Cervicothoracic Spine: A Review of Current Applications
}

\author{
JIAN SHEN, MD, PHD ${ }^{1}$; ELIAS SHAAYA, MD ${ }^{2}$; JUNSEOK BAE, MD ${ }^{3}$; AND ALBERT E. TELFEIAN, MD, PHD ${ }^{2}$ \\ ${ }^{I}$ Shen-Spine, New York, New York; ${ }^{2}$ Department of Neurosurgery, Rhode Island Hospital, The Warren Alpert Medical School of Brown University, Providence, Rhode \\ Island; ${ }^{3}$ Department of Neurosurgery, Wooridul Spine Hospital, Seoul, South Korea
}

\begin{abstract}
Background: Endoscopic spine surgery in the cervicothoracic spine is generating continued interest in a rapidly evolving field. The authors present 4 techniques for fully endoscopic cervical spine surgery: (1) posterior cervical unilateral laminectomy and bilateral decompression, (2) posterior cervical foraminotomy, (3) anterior cervical discectomy, and (4) anterior transcorporal discectomy. Two techniques for fully endoscopic thoracic spine surgery are also presented: (1) posterior thoracic unilateral laminectomy and bilateral decompression and (2) transforaminal thoracic endoscopic discectomy and foraminotomy.

Methods: We describe 6 different surgical approaches and review the relevant literature about each technique.

Results: The clinical application of endoscopic spine surgery techniques has evolved over the past 40 years. Recent data suggest comparable outcomes to other procedures and perhaps fewer complications and quicker recovery when these techniques are used in the cervical and thoracic spine. Significant variability exists in these approaches depending on the goal of canal decompression, root decompression, and the site of the pathology.

Conclusions: Each endoscopic approach in the cervicothoracic spine has its technical nuances, outcomes, advantages, and disadvantages, making fully endoscopic cervicothoracic spine surgery an exciting and growing field.
\end{abstract}

Special Issue

Keywords: endoscopic discectomy, transforaminal, TESSYS, radiculopathy, cervical, thoracic

\section{INTRODUCTION}

Cervical disc herniation and cervical stenosis are common and can present as radiculopathy or myelopathy. ${ }^{1}$ Traditional surgical approaches include anterior cervical discectomy and fusion, posterior cervical foraminotomy, and posterior laminectomy with or without fusion. These approaches have been exhaustively studied and validated. ${ }^{2-6}$ Thoracic disc herniations represent a host of surgical challenges, as each access corridor presents anatomical hazards. Open surgical approaches for the treatment of thoracic disc herniations and thoracic stenosis, from medial to lateral, include laminectomy, transpedicular, costotransversectomy, and transthoracic. The goal of each of these approaches is to achieve adequate decompression with little to no manipulation of the spinal cord.

Fully endoscopic cervicothoracic spine surgery is typically cervicothoracic spine surgery performed through a working-channel endoscope. Unlike open or microendoscopic cervicothoracic spine surgery, the tubular retractor is so small that the operative field is not visible even with loupes or microscope. Biportal fully endoscopic spine surgery, as the name implies, utilizes 2 ports: a port for surgical instruments and a port for an endoscopic camera. Endoscopic approaches to the cervicothoracic spine have been reported in the literature and found to be safe and effective. ${ }^{7,8}$

Here, we review 4 techniques for fully endoscopic cervical spine surgery: (1) posterior cervical unilateral laminectomy and bilateral decompression, (2) posterior cervical foraminotomy, (3) anterior cervical discectomy, and (4) anterior transcorporal discectomy. We also review 2 techniques for fully endoscopic thoracic spine surgery: (1) posterior thoracic unilateral laminectomy and bilateral decompression and (2) transforaminal thoracic endoscopic discectomy and foraminotomy.

\section{METHODS}

\section{Surgical Procedures: Cervical}

\section{Posterior Cervical Unilateral Laminectomy and Bilateral Decompression}

This technique is indicated for the treatment of cervical stenosis. The patient is positioned prone in a Mayfield head holder on hip and chest bolsters. The procedure is performed under general anesthesia, and somatosensory-evoked potential monitoring is typically used. Figure 1 depicts a case example. The joimax 


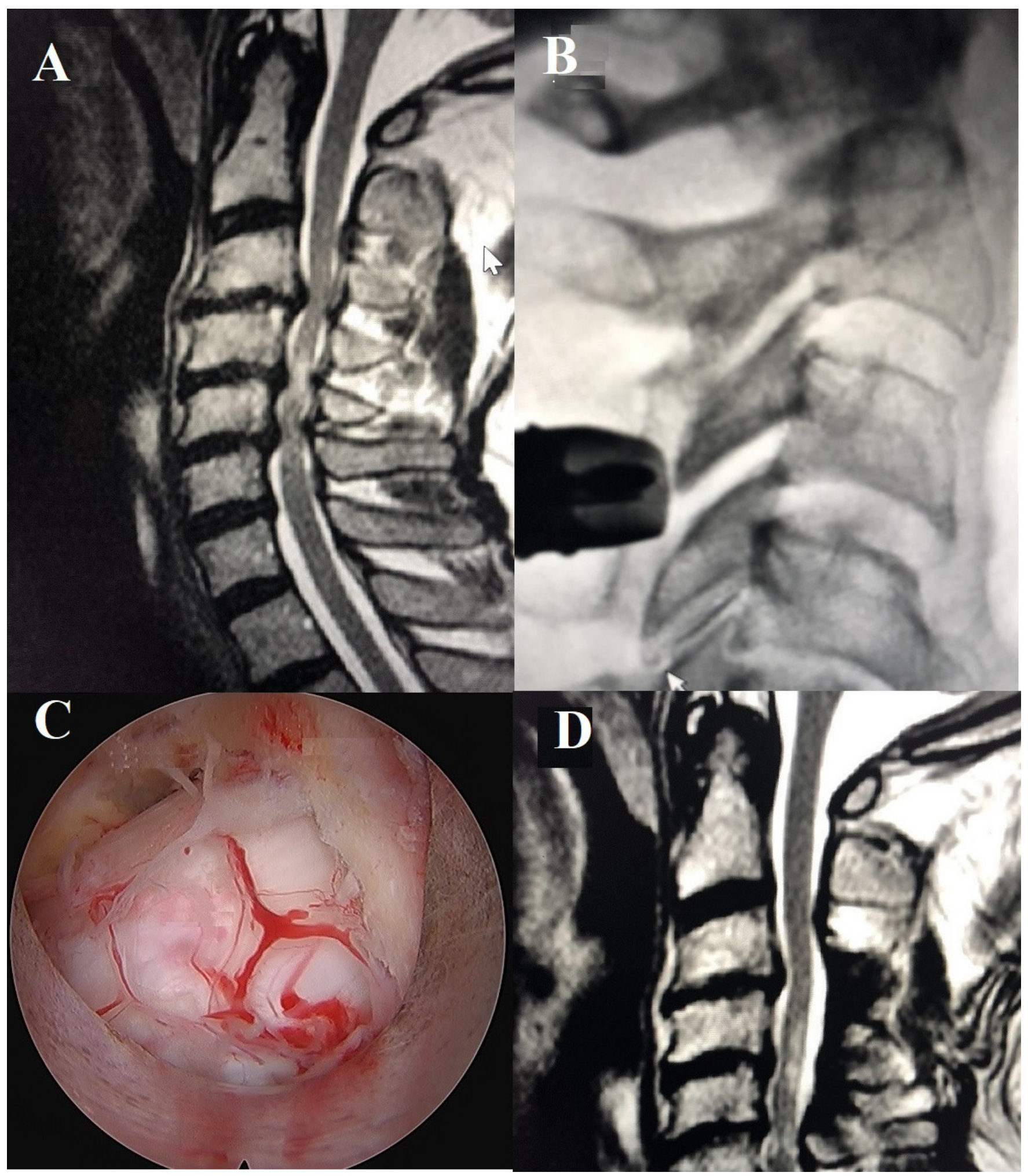

Figure 1. Posterior cervical unilateral laminectomy and bilateral decompression. (A) Preoperative T2-weighted sagittal magnetic resonance image demonstrates severe spinal cord stenosis from C3-6 and cord myelomalacia. (B) Lateral fluoroscopic image depicts the cannulated beveled tubular retractor with the Delta working-channel endoscope and Shrill drill at the spinolaminar junction of C3-4. (C) Endoscopic camera view of the bilaterally decompressed thecal sac. (D) Postoperative T2-weighted sagittal magnetic resonance image demonstrating the resolution of the cervical stenosis after the C3-6 endoscopic laminectomy. 
iLESSYS Delta endoscopic system is used for the procedure. A $1-\mathrm{cm}$ incision is made through the skin, $1 \mathrm{~cm}$ lateral to the midline. Using intermittent fluoroscopic guidance, sequential dilators and a final 10-mm beveled tubular retractor are placed at the junction of the spinous process and the lamina. The A workingchannel endoscope with a $10-\mathrm{mm}$ outer diameter and a 6-mm working channel is then placed, and a high-speed Shrill drill is used to complete the subspinous process laminectomy. An endoscopic micro Kerrison rongeur is used to remove the ligamentum flavum and complete the laminectomy or laminotomy. Figure 1 demonstrates preoperative and postoperative magnetic resonance images, intraoperative fluoroscopic images, and endoscopic camera views of a multilevel posterior cervical unilateral laminectomy for bilateral decompression. As demonstrated in Figure 1, a single incision can be used for up to 3 levels in multilevel cases. Sequential dilation is performed separately for each level through the same incision. The technique for bilateral decompression is an "over-the-top" decompression in which the endoscopic drill is used to remove the proximal hemilamina and then, through a subspinous process corridor, the contralateral hemilamina. The decompression is complete when the bilateral ligamentum flavum is removed and the pulsatile dura is seen. A full description of the technique is available from Shen et $\mathrm{al}^{9}$ and Ahn. ${ }^{10}$

\section{Posterior Cervical Foraminotomy}

This indication is for the treatment of cervical radiculopathy that results from foraminal bony narrowing or a paracentral or foraminal disc herniation. The patient is positioned prone in a Mayfield head holder on hip and chest bolsters. The procedure is performed under general anesthesia. Figure 2 depicts a case example. The joimax iLESSYS Pro endoscopic system with a 7.3-mm outer diameter is used for the procedure. Percutaneous entry is established through a $1-\mathrm{cm}$ skin incision $1 \mathrm{~cm}$ lateral to the midline. Using intermittent fluoroscopic guidance, a 3.5-in 18-gauge needle is advanced, and the tip is placed at the junction of the inferior lamina and facet complex. A Kirschner wire (K-wire) is placed, the needle is removed, and sequential dilators are placed at the superior part of the inferior lamina at the facet junction, followed by a 7-mm beveled tubular retractor. The Shrill diamond drill is used to perform the foraminotomy, which is completed with the endoscopic Micro Kerrison Punch. The foraminotomy is completed when the exiting nerve root can be visualized, and the decompression is carried lateral to the medial wall of the inferior pedicle. Discectomy can be performed, when applicable, after the foraminotomy. The beveled tubular retractor is used to retract the nerve by turning the bevel so that the open side of the retractor faces away from the canal. In this "turn-to-retract" method, an endoscopic grasper is used to remove the disc herniation. Figure 2 presents the preoperative images, intraoperative fluoroscopic images, and endoscopic camera views from an endoscopic posterior cervical foraminotomy case. A full description of the technique is available from Shen et $\mathrm{al}^{9}$ and Ahn. ${ }^{10}$

\section{Anterior Cervical Discectomy}

Surgery is indicated for the treatment of cervical radiculopathy or myelopathy caused by a herniated cervical disc. The patient is positioned supine, and the procedure can be performed under general anesthesia (intraoperative neuromonitoring is recommended) or without anesthesia if the patient is comfortable with preoperative palpation of the anterior vertebral body. Performing the surgery with conscious sedation using midazolam and fentanyl offers the surgeon the advantage of real-time neuromonitoring. Figure 3 depicts an example case. Fluoroscopy is performed to determine the appropriate level. After palpating the carotid pulsation, the trachea and esophagus are gently displaced to the contralateral side. The access needle is inserted into the disc space under the lateral fluoroscopic projection and advanced into the posterior disc space. A guidewire is inserted through the needle, and a 5-mm skin incision is made medial to the sternocleidomastoid muscle. After sequential dilators are placed, the final tubular retractor is introduced down to the posterior vertebral body. The 3.9-mm endoscope (CESSYS, joimax $\mathrm{GmbH}$, Germany) is introduced into the tubular retractor with a $4.5-\mathrm{mm}$ outer diameter. The discectomy is a targeted procedure with dilators placed through the disc, targeting the herniation at the disc's posterior edge. The herniated fragment can be removed with endoscopic grasping forceps. In cases of foraminal disc herniation, foraminal decompression by resection of the uncinate process may be required. The osteophyte and posterior part of the uncinated process can be removed with an endoscopic reamer or diamond drill, giving the surgeon access to the foraminal area for the removal of a herniated disc and direct visualization of the nerve root. After decompression, dural pulsation and the decompressed neural structure can be observed. A full description of the technique is available from Shen et $\mathrm{al}^{9}$ and Ahn. ${ }^{10}$ 


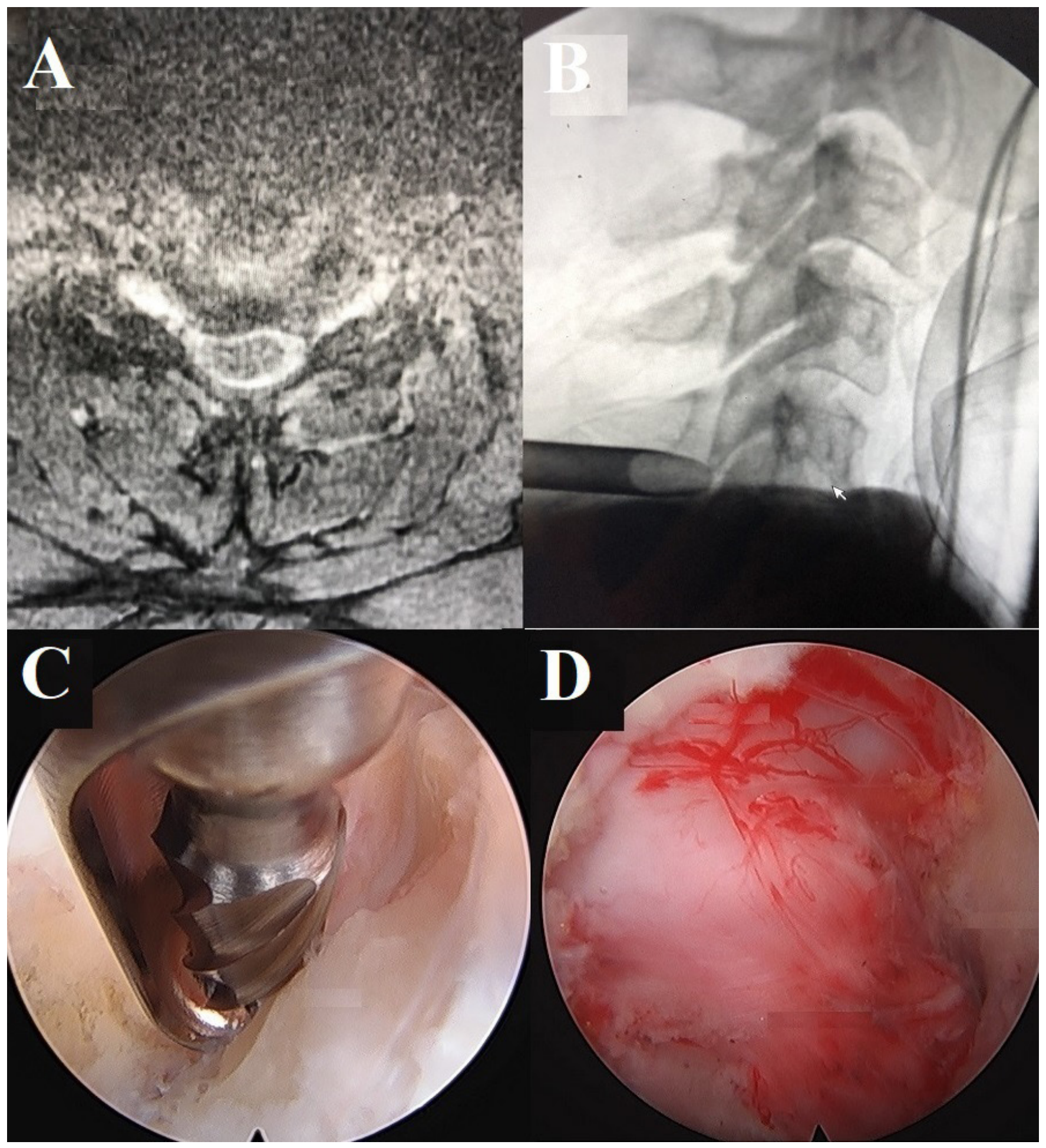

Figure 2. Posterior cervical foraminotomy. (A) Preoperative T2 axial magnetic resonance image illustrating right C5-6 foraminal stenosis. (B) Lateral fluoroscopic view demonstrating the position of the beveled tubular retractor on the laminar-facet junction at C5-6. (C) Endoscopic camera views of the laminar-facet junction and the Shrill drill removing the superior lateral edge of the C6 lamina and (D) the decompressed C6 nerve root.

\section{Anterior Transcorporal Discectomy}

This technique is indicated for the removal of a soft sequestered disc fragment when the surgeon is concerned about violating the otherwise normal nucleus pulposa. The patient is positioned supine, and the procedure is performed under general anesthesia. Figure 4 depicts an example case. The approach is similar to the anterior cervical discectomy technique: a $1.5-\mathrm{cm}$ 


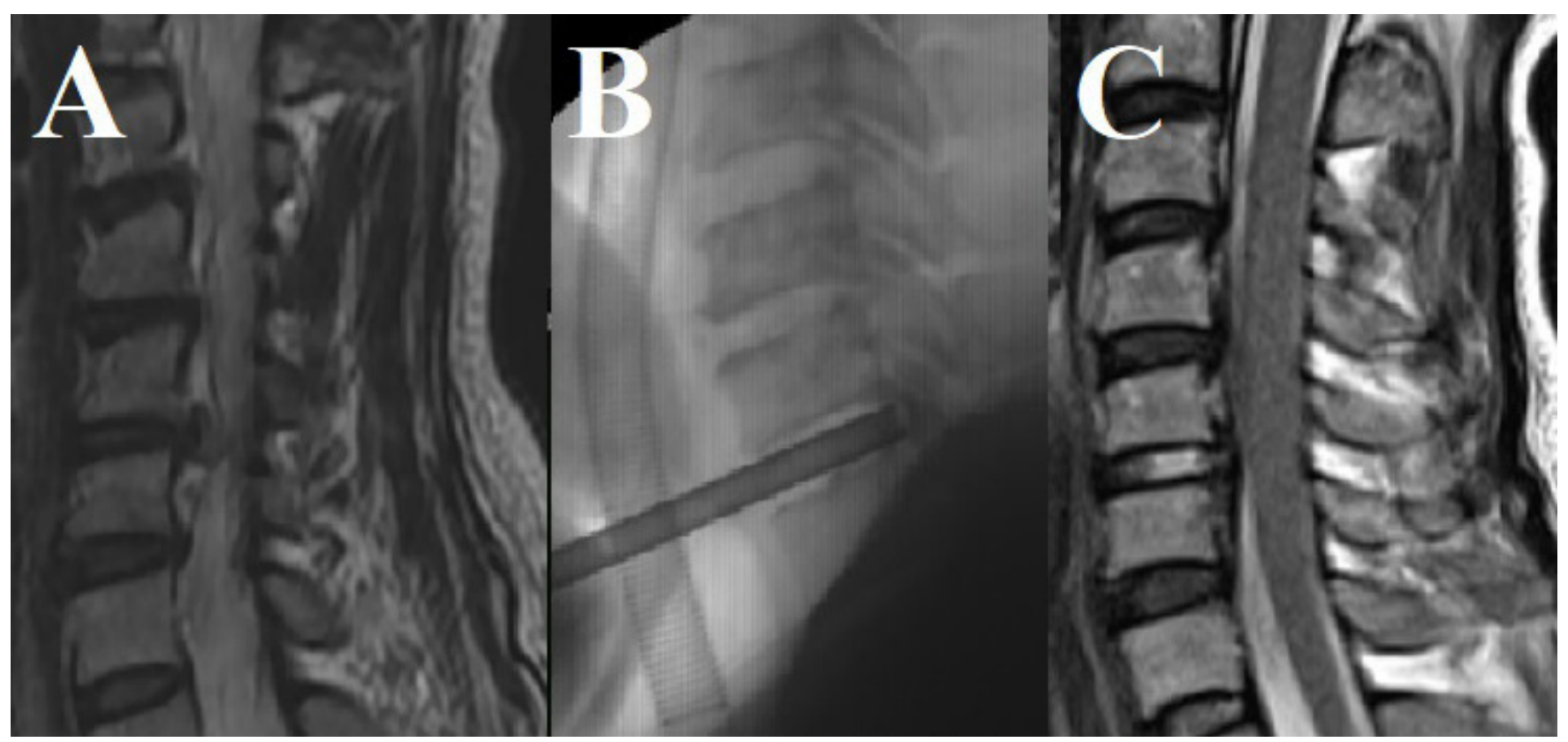

Figure 3. Anterior cervical discectomy. (A) Preoperative T2 magnetic resonance image showing foraminal disc herniation at C5-6 on the left side. (B) Lateral fluoroscopic image demonstrating the tubular retractor in the disc space. (C) Postoperative T2 sagittal magnetic resonance image showing complete decompression.

incision is made just medial to the sternocleidomastoid muscle, and blunt finger dissection is performed using a standard Smith-Robinson approach. First, a 13-mm Matrix tubular retractor is placed over the upper vertebral body, and a guide pin is placed into the upper vertebral body with a planned trajectory toward the disc herniation (head light and loupes are used). Next, a 6.5-mm cannulated power drill is used to drill over the guide pin, and a Shrill drill is used to finish drilling a channel through the superior vertebra to allow placement of the tubular retractor with its distal opening at the junction of the inferior endplate of the superior vertebral body and the posterior longitudinal ligament. The TESSYS working-channel endoscope (6.3-mm outer diameter) is used, and instead of entering the disc with the tubular retractor, drilling is performed using intermittent fluoroscopic guidance through the superior vertebral body to safely target the disc pathology. The channel created allows access to the herniated ventral disc fragment without violating the anterior portion of the disc. Because this is a fragmentectomy procedure for a soft sequestered fragment, the endpoint of the

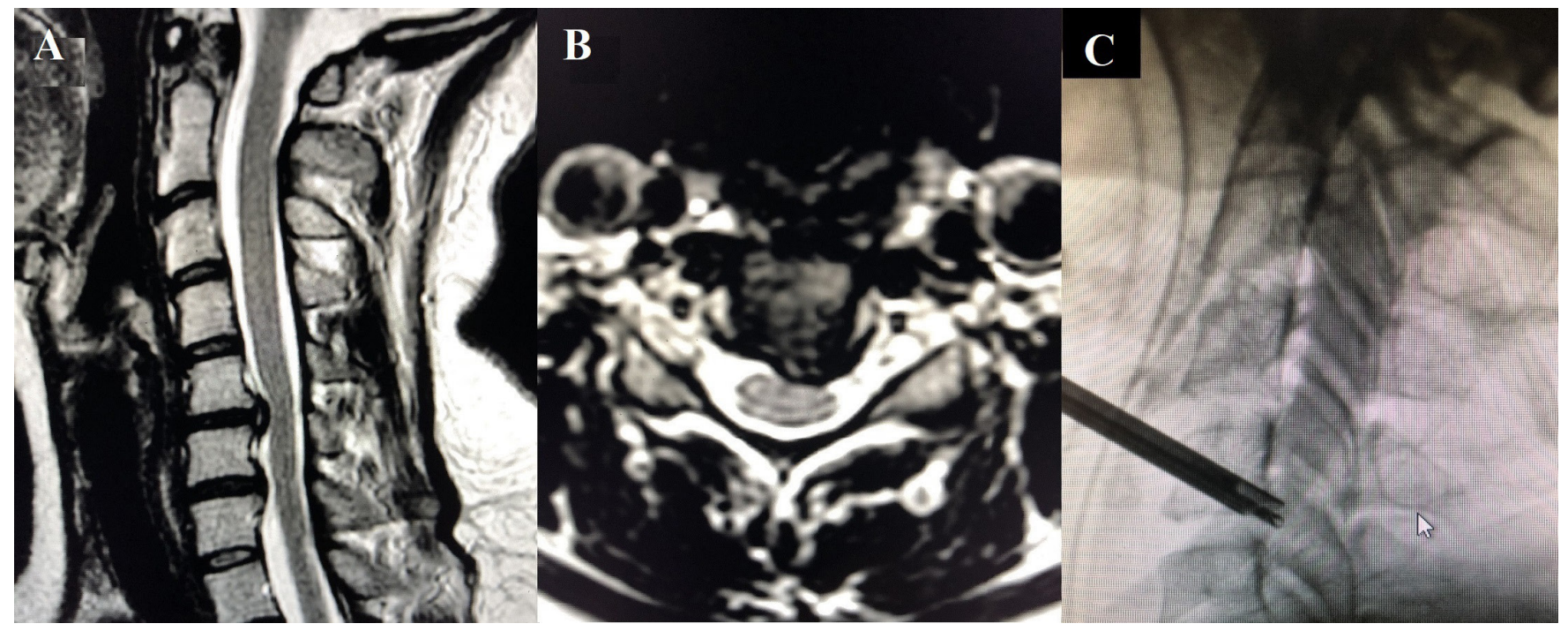

Figure 4. Anterior transcorporal discectomy. (A) Preoperative T2 sagittal and (B) axial magnetic resonance images of a C5-6 herniated disc. (C) Lateral fluoroscopic image of the TESSYS endoscope and tubular retractor placed through the body of C5. 
surgery is determined when the fragment is removed. A full description of the technique is available from Shen et $\mathrm{al}^{9}$ and $\mathrm{Ahn} .{ }^{10}$

\section{Surgical Procedures: Thoracic}

\section{Posterior Thoracic Unilateral Laminectomy and Bilateral Decompression}

The indication for this surgery is the treatment of thoracic spinal canal stenosis. The patient is positioned prone, and surgery is performed with the patient awake under conscious sedation. Figure 5 depicts an example case. A $1-\mathrm{cm}$ incision is made through the skin $1 \mathrm{~cm}$ lateral to the midline. Using intermittent fluoroscopic guidance, sequential dilators and a final 10-mm beveled tubular retractor are placed at the junction of the spinous process and lamina. The joimax Delta endoscope with a $10-\mathrm{mm}$ outer diameter and a 6-mm working channel is then placed, and a high-speed Shrill drill is used to complete the subspinous process laminectomy. An endoscopic micro Kerrison rongeur is used to remove the ligamentum flavum and to finish the laminectomy/ laminotomy. This procedure is similar to the posterior cervical unilateral laminectomy and bilateral decompression, but the authors feel that thoracic decompression can be done with the patient awake. Local anesthesia and sedation are sufficient pain control. Hemostasis is controlled with a radiofrequency probe. In cases in which the surgeon feels additional hemostatic control is necessary, a hemostatic matrix can be applied to the operative field, and a drain can even be placed through the endoscope and removed the next postoperative day. A full description of this technique is available from Shen et al. ${ }^{11}$

\section{Transforaminal Thoracic Endoscopic Discectomy and Foraminotomy}

This technique is best suited for thoracic radiculopathy. The patient is positioned prone, and surgery is performed with the patient awake under local anesthesia and conscious sedation. Figure 6 depicts an example case. Preoperatively, the trajectory for the transforaminal approach angle is measured on the axial magnetic resonance images, making sure to avoid the ribs and pleura. Percutaneous entry is established 5 to $7 \mathrm{~cm}$ off the midline. Using intermittent fluoroscopic guidance, a 15-cm 18-gauge needle is advanced and placed at the superior facet of the surgical level. Subsequently, the sequential dilators are passed through the soft tissue and docked on the ventral portion of the ipsilateral superior facet. The beveled tubular retractor is placed, and the joimax (Irvine, USA) TESSYS endoscopic system with a $25^{\circ}$ viewing angle is used. A high-speed drill (Joimax Shrill, 3.5-mm diamond abrasor) is then used to enlarge the neural foramen (foraminoplasty) by drilling the ventral aspect of the superior facet, the proximal part of the caudal pedicle, and part of the rib head under direct visualization. All surgical instruments are introduced under continuous irrigation and with direct visualization through the intraendoscopic working channel. The facet, pedicle, disc, and ligamentum flavum are visualized, and ventral epidural decompression is accomplished by slowly advancing the endoscope into the ventral epidural space under direct visualization and removing any disc extrusion without manipulating or retracting the spinal cord. Treating calcified discs with this approach is recommended only for advanced practitioners. In addition to the risk of spinal cord injury, spinal cord compression could persist after a large decompression secondary to an "eggshell" ridge of calcification. A full description of this technique is available from Wagner et al. ${ }^{12}$

\section{Master Technique (Technical Tips)}

There are several "master techniques" for performing successful endoscopic cervicothoracic surgery.

\section{Targeting}

Endoscopic visualization can be a great advantage because the camera is located at the site of the pathology, but endoscopic cameras have a limited field of view. If the endoscope is not exactly at the target pathology, surgery will not be successful. Utilizing anteroposterior and lateral fluoroscopy to confirm the exact position of the tubular retractor is a critical step before performing any endoscopic decompression.

\section{Safety}

Many surgeons new to endoscopic procedures prefer to perform them under general anesthesia because they are anxious about the new technique. Awake endoscopic surgery is recommended, whenever possible, to those surgeons because immediate feedback from the patient can make the difference in preventing irreversible nerve or spinal cord damage. For posterior cervical endoscopic surgery, which we do not recommend performing awake, fluoroscopic confirmation of the endoscope position and neuromonitoring are even more critical. 


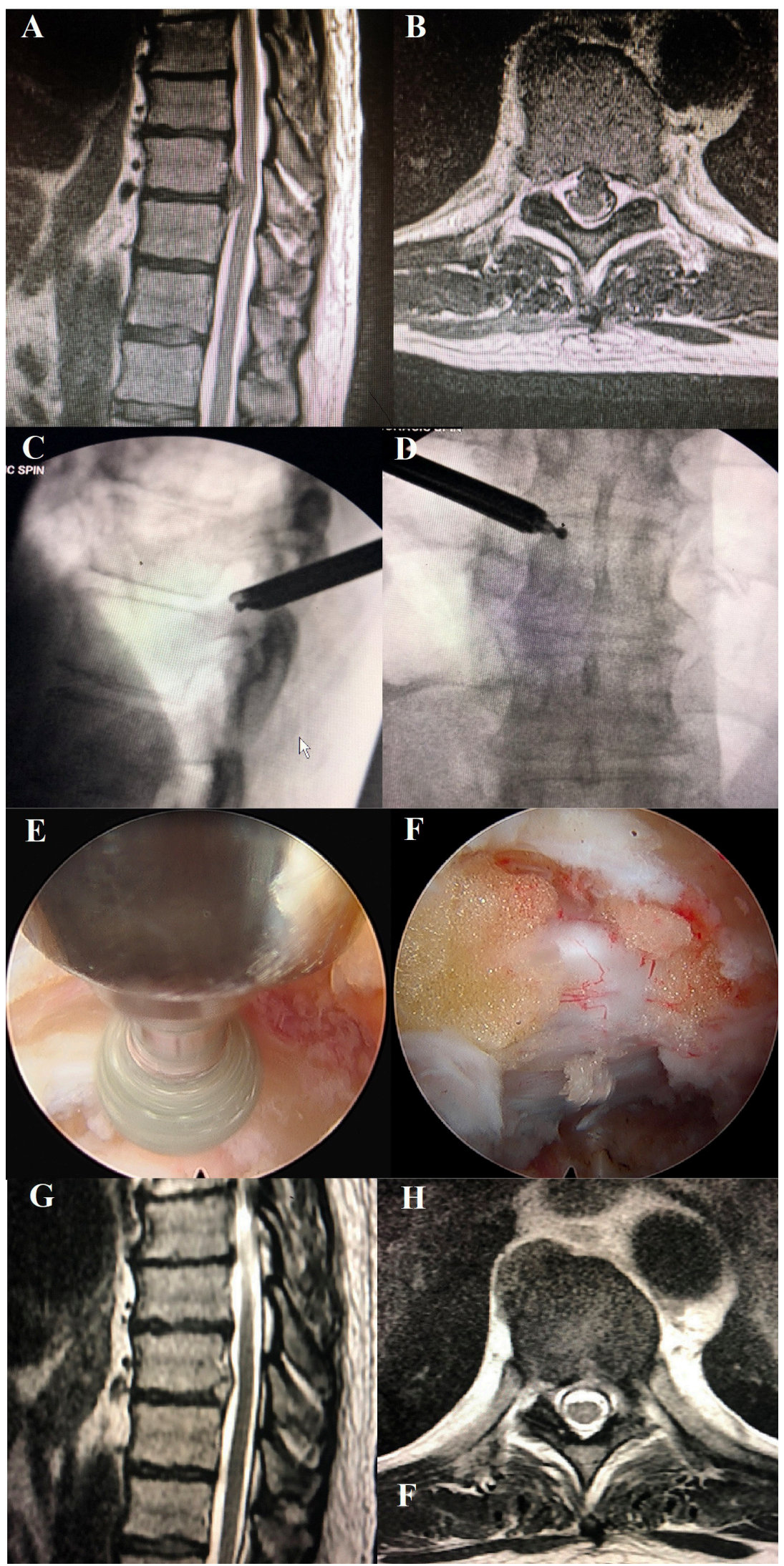

Figure 5. Posterior thoracic unilateral laminectomy and bilateral decompression. (A) Sagittal and (B) axial T2-weighted magnetic resonance images of a T9-10 disc herniation and severe canal stenosis and increased cord signal. (C) Lateral and (D) anteroposterior fluoroscopic images of the tubular retractor, working-channel endoscope with 10-mm outer diameter, and endoscopic drill used for the laminectomy procedure. (E) Intraoperative endoscopic image showing decompressed thecal sac after drilling. 


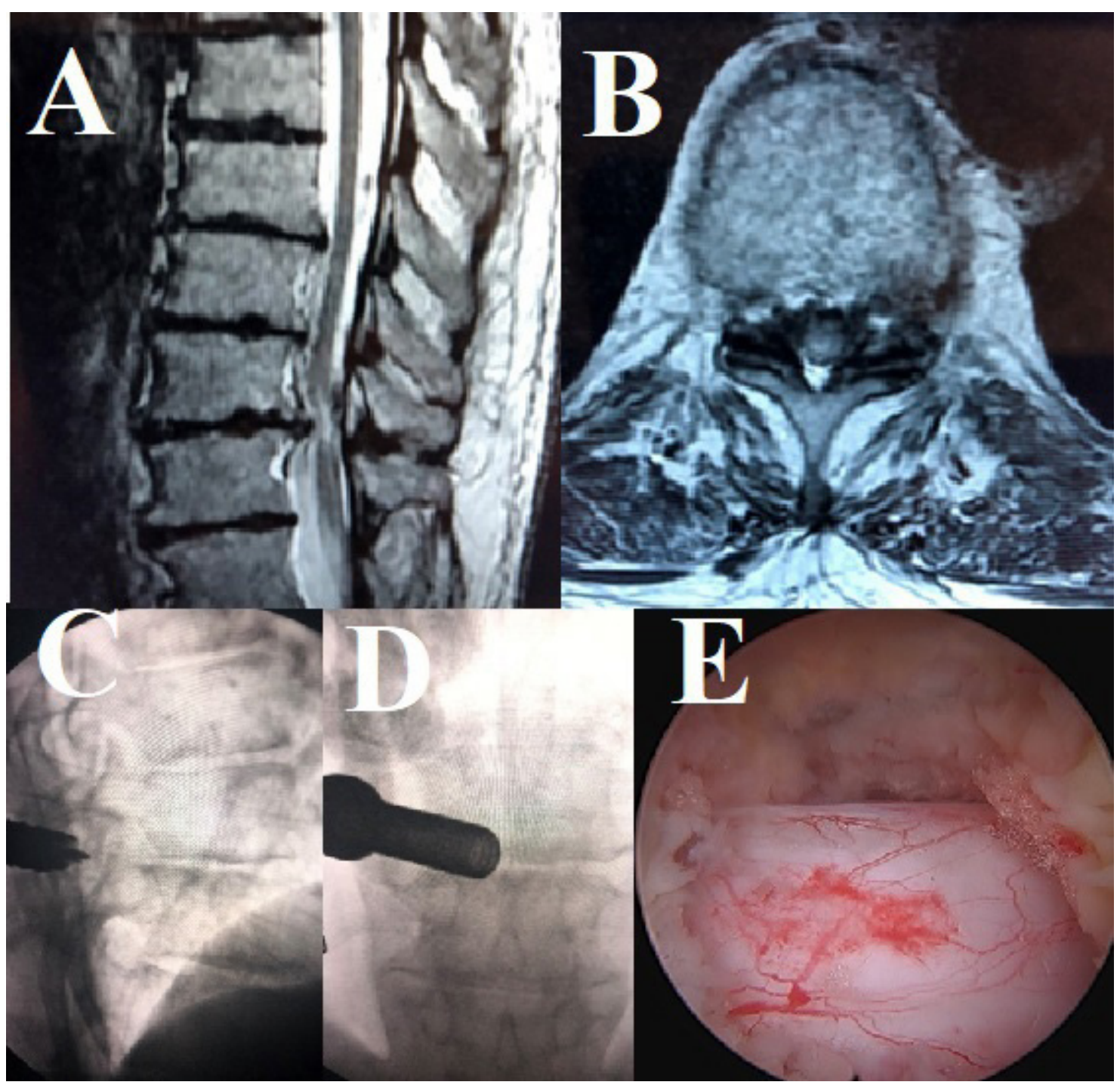

Figure 6. Transforaminal thoracic endoscopic discectomy and foraminotomy. (A) Sagittal and (B) axial T2-weighted magnetic resonance image demonstrating a T9-10 central disc extrusion and cord compression. (C) Intraoperative lateral and (D) anteroposterior fluoroscopic images demonstrating the placement of the beveled tubular retractor, endoscope, and high-speed drill; the drill is removing the ventral portion of the superior articular process. (E) Intraoperative endoscopic image demonstrating drilling of the ventral portion of the superior articular process (foraminoplasty), taken at the same time as $6 \mathrm{C}$ and $6 \mathrm{D}$. (F) Intraoperative endoscopic image of the thecal sac after drilling of the superior articular process. (G) Postoperative T2-weighted sagittal and (H) axial magnetic resonance images of the decompressed spinal cord.

\section{Learning Curve}

Cervicothoracic endoscopic cases may be a new challenge even for surgeons familiar with endoscopic spine techniques. Cadaver labs are a good opportunity to practice these techniques. Finding a mentor to observe or proctor cases is an excellent way to get over the hurdle of approaching these surgeries.

\section{Planning}

For thoracic cases, preoperative planning based on the axial magnetic resonance images is critical for determining the starting point and entry angle to avoid the ribs and pleura. The superior articular process is relatively large in the thoracic spine, so the surgeon should expect added time for bony decompression.

\section{Thoracolumbar}

Thoracolumbar cases are similar to lumbar endoscopic cases in that the procedure is performed from a more medial starting point.

\section{Nondegenerative Pathology}

Endoscopically approaching tumors, cement, and hardware is possible in the cervicothoracic spine but should be considered only in advanced-level cases. 


\section{Hemostasis}

A radiofrequency probe is used for hemostasis. Highdefinition endoscopic imaging allows for excellent visualization of individual bleeding vessels. For bleeding that is refractory to coagulation, a hemostatic matrix can be injected and subsequently suction evacuated.

\section{Safety in Posterior Cervical Foraminotomy}

Drilling the medial third of the pedicle and corpus ventral to the spinal cord and exiting the nerve root by 3 to $5 \mathrm{~mm}$ creates space for an efficient discectomy and uncus decompression while still preserving the stability of the spinal segment. ${ }^{13}$

\section{DISCUSSION}

Indications for cervicothoracic endoscopic spine surgery are essentially the same as those for traditional anterior and posterior cervicothoracic techniques. Most patients undergoing endoscopic cervicothoracic procedures are seeking out the most minimally invasive surgical procedure available.

Anterior cervical endoscopic approaches, described here, are minimally invasive but do not include fusion. It is reasonable to question whether the results of anterior endoscopic discectomy can compare to the very successful outcomes of anterior cervical discectomy and fusion. A retrospective study of 187 patients undergoing anterior endoscopic cervical discectomy by Parihar et al showed a decrease in visual analog scale scores for arm and neck pain (from 6.7 to 1.7 and from 3.2 to 1.1 , respectively), with only 2 patients requiring a second procedure and a mean follow-up of 29 months. ${ }^{14}$ This same series showed that, despite the lack of fusion and placement of an intervertebral spacer, cervical lordosis improved. However, reports of cervical lordosis in the literature have varied, with some suggesting that anterior cervical discectomy and fusion are superior in maintaining proper cervical alignment. Results have ranged from improved to unchanged and worsened lordosis. ${ }^{15,16}$ However, these reports also show improved clinical outcomes despite the different cervical alignments seen on imaging. 15,16 Although fusion would theoretically improve or restore cervical lordosis, fusion does not come without a cost; the risks inherent with fusion surgery, such as pseudarthroses and adjacent segment disease, are avoided with endoscopic anterior cervical surgery.

Likewise, successful results have been reported in posterior endoscopic cervical techniques. A recent study by Wan et al reports their experience with posterior full endoscopic cervical discectomy under local anesthesia. ${ }^{17}$ Twenty-five patients underwent the procedure with 24 achieving resolution of symptoms, and no major complications were reported. ${ }^{17}$ Another study looked at strength recovery after posterior endoscopic discectomy and reported a $95 \%$ rate of strength recovery with $86 \%$ experiencing full resolution of weakness at the 1 year follow-up. ${ }^{18}$ Proponents of endoscopic posterior approaches have argued that they minimize postoperative pain due to the lack of muscle stripping, avoid blood loss, reduce operative time, and shorten hospital stays.

Full endoscopic surgery for thoracic pathology was reviewed by Gibson et al in 2021. The review included papers from 2006 to 2020 comprising 17 patient series, 1 cohort study, and 13 case reports with 1 to 3 patients. ${ }^{8}$ In 460 patients, complications reported were dural tears $(2 \%)$, transient paresthesia $(2 \%)$, revision surgery $(1.5 \%)$, neurologic injury $(0.6 \%)$, and epidural hematoma $(0.6 \%) .{ }^{19-32}$ The review concluded that there was clear evidence for a lower complication rate and shorter hospital stay with endoscopic thoracic spine surgery approaches versus open approaches. Moreover, there were no reported cases in the endoscopic experience of wrong-level surgery, vertebral column instability, cerebrospinal fluid leaks/pleural fistulas, thoracic viscera injury, or intercostal neuralgia. ${ }^{8}$

\section{CONCLUSION}

Fully endoscopic cervicothoracic spine surgery is an emerging minimally invasive surgical option for the treatment of symptomatic cervicothoracic radiculopathy and myelopathy. Advantages include the highdefinition visualization of the surgical pathology and the reduction of tissue trauma. Anterior cervical endoscopic discectomy techniques offer additional advantages, including preservation of movement at that level, lack of fusion, and minimal to no manipulation of nerve roots. The main disadvantages include the steep learning curve of endoscopic techniques for surgeons accustomed to traditional open approaches, the limited direct field of view, and the narrow working channel. Larger long-term prospective trials with a control arm are still needed to tease out the true potential and shortcomings of endoscopic approaches to the cervicothoracic spine as compared to traditional techniques. Additional studies on outcomes and technical nuances in endoscopic anterior and posterior cervical foraminotomy and discectomy, ${ }^{33-38}$ transcorporal discectomy, ${ }^{39}$ cervical laminectomy, ${ }^{40}$ thoracic laminectomy, ${ }^{19,28,41,42}$ and transforaminal thoracic decompression $^{23,43}$ have been published and offer excellent 
insights into these techniques that focus on the individual procedures.

\section{REFERENCES}

1. Woods BI, Hilibrand AS. Cervical radiculopathy: epidemiology, etiology, diagnosis, and treatment. J Spinal Disord Tech. 2015;28(5):E251-9. doi:10.1097/BSD.0000000000000284.

2. Martins AN. Anterior cervical discectomy with and without interbody bone graft. J Neurosurg. 1976;44(3):290-295. doi:10.3171/jns.1976.44.3.0290.

3. Rosenørn J, Hansen EB, Rosenørn MA. Anterior cervical discectomy with and without fusion. A prospective study. J Neurosurg. 1983;59(2):252-255. doi:10.3171/jns.1983.59.2.0252.

4. Bohlman HH, Emery SE, Goodfellow DB, Jones PK. Robinson anterior cervical discectomy and arthrodesis for cervical radiculopathy. Long-term follow-up of one hundred and twentytwo patients. J Bone Joint Surg Am. 1993;75(9):1298-1307. doi:10.2106/00004623-199309000-00005.

5. Villavicencio AT, Pushchak E, Burneikiene S, Thramann JJ. The safety of instrumented outpatient anterior cervical discectomy and fusion. Spine J. 2007;7(2):148-153. doi:10.1016/j. spinee.2006.04.009.

6. Rhee JM, Basra S. Posterior surgery for cervical myelopathy: laminectomy, laminectomy with fusion, and laminoplasty. Asian Spine J. 2008;2(2):114-126. doi:10.4184/asj.2008.2.2.114.

7. Yang J-. S, Chu L, Chen L, Chen F, Z-Y K, Deng Z-. L. Anterior or posterior approach of full-endoscopic cervical discectomy for cervical intervertebral disc herniation? A comparative cohort study. Spine. 2014;39:1743-1750. doi:10.1097/ BRS.0000000000000508.

8. Gibson RDS, Wagner R, Gibson JNA. Full endoscopic surgery for thoracic pathology: an assessment of supportive evidence. EFORT Open Rev. 2021;6(1):50-60. doi:10.1302/20585241.6.200080.

9. Shen J, Telfeian AE, Shaaya E, Oyelese A, Fridley J, Gokaslan ZL. Full endoscopic cervical spine surgery. J Spine Surg. 2020;6(2):383-390. doi:10.21037/jss.2019.10.15.

10. Ahn Y. The current state of cervical endoscopic spine surgery: an updated literature review and technical considerations. Expert Rev Med Devices. 2020;17(12):1285-1292. doi:10.1080/174 34440.2020 .1853523$.

11. Shen J, Telfeian AE. Fully endoscopic $360^{\circ}$ decompression surgery for thoracic spinal stenosis: technical note and report of 8 cases. Pain Physician. 2020;23(6):E659-E663. doi:10.36076/ ppj.2020.23.E659.

12. Wagner R, Telfeian AE, Iprenburg M, et al. Transforaminal endoscopic foraminoplasty and discectomy for the treatment of a thoracic disc herniation. World Neurosurg. 2016;90:194-198. doi:10.1016/j.wneu.2016.02.086.

13. Kim HS, PH W, Lee YJ, et al. Safe route for cervical approach: partial pediculotomy, partial vertebrotomy approach for posterior endoscopic cervical foraminotomy and discectomy. World Neurosurg X. 2020;140:e273-e282. doi:10.1016/j. wneu.2020.05.033.

14. Parihar VS, Yadav N, Ratre S, Dubey A, Yadav YR. Endoscopic anterior approach for cervical disc disease (disc preserving surgery). World Neurosurg. 2018;115:e599-e609. doi:10.1016/j. wneu.2018.04.107.
15. Haden N, Latimer M, Seeley HM, Laing RJ. Loss of intervertebral disc height after anterior cervical discectomy. Br J Neurosurg. 2005;19(6):469-474. doi:10.1080/02688690500495109.

16. Kim CH, Shin K-H, Chung CK, Park SB, Kim JH. Changes in cervical sagittal alignment after single-level posterior percutaneous endoscopic cervical diskectomy. Global Spine J. 2015;5(1):31-38. doi:10.1055/s-0034-1395423.

17. Wan Q, Zhang D, Li S, et al. Posterior percutaneous fullendoscopic cervical discectomy under local anesthesia for cervical radiculopathy due to soft-disc herniation: a preliminary clinical study. J Neurosurg Spine. 2018;29(4):351-357. doi:10.3171/2018. 1.SPINE17795.

18. Lee U, Kim CH, Chung CK, et al. The recovery of motor strength after posterior percutaneous endoscopic cervical foraminotomy and discectomy. World Neurosurg. 2018;115:e532-e538. doi:10.1016/j.wneu.2018.04.090.

19. An B, Li X-C, Zhou C-P, et al. Percutaneous full endoscopic posterior decompression of thoracic myelopathy caused by ossification of the ligamentum flavum. Eur Spine J. 2019;28(3):492-501. doi:10.1007/s00586-018-05866-2.

20. Bae J, Chachan S, Shin S-H, Lee S-H. Percutaneous endoscopic thoracic discectomy in the upper and midthoracic spine: a technical note. Neurospine. 2019;16(1):148-153. doi:10.14245/ ns.1836260.130.

21. Bae J, Chachan S, Shin S-H, Lee S-H. Transforaminal endoscopic thoracic discectomy with foraminoplasty for the treatment of thoracic disc herniation. J Spine Surg. 2020;6(2):397-404. doi:10.21037/jss.2019.11.19.

22. Cheng X-. K, Chen B. Percutaneous endoscopic thoracic decompression for thoracic spinal stenosis under local anesthesia. World Neurosurg. 2020;139:488-494. doi:10.1016/j. wneu.2020.04.199.

23. Guo C, Zhu D, Kong Q, et al. Transforaminal percutaneous endoscopic decompression for lower thoracic spinal stenosis. World Neurosurg. 2019;128:e504-e512. doi:10.1016/j.wneu.2019.04.186.

24. Lee HY, Lee S-. H, Kim D-. Y, Kong BJ, Ahn Y, Shin S-. W. Percutaneous endoscopic thoracic discectomy: posterolateral transforaminal approach. J Korean Neurosurg Soc. 2006;40:58-62.

25. Lee SH. Posterolateral endoscopic thoracic discectomy: transforaminal approach. nsmc. 2018;1(2):20. doi:10.15562/nsmc. v1i2.98.

26. Li ZZ, Zhao HL, Cao Z, Shang WL, Hou SX. Technical notes and clinical efficacy analysis of full-endoscopic thoracic discectomy via transforaminal approach. Zhonghua Yi Xue Za Zhi. 2020;100(4):279-285. doi:10.3760/cma.j.issn.0376-2491.2020.04. 008.

27. Nie H-F, Liu K-X. Endoscopic transforaminal thoracic foraminotomy and discectomy for the treatment of thoracic disc herniation. Minim Invasive Surg. 2013;2013:264105. doi:10.1155/2013/264105.

28. Ruetten S, Hahn P, Oezdemir S, et al. Full-endoscopic uniportal decompression in disc herniations and stenosis of the thoracic spine using the interlaminar, extraforaminal, or transthoracic retropleural approach. J Neurosurg Spine. 2018;29(2):157-168. doi:10.3171/2017.12.SPINE171096.

29. Ruetten S, Hahn P, Oezdemir S, Baraliakos X, Godolias G, Komp M. Operation of soft or calcified thoracic disc herniations in the full-endoscopic uniportal extraforaminal technique. Pain Physician. 2018;21(4):E331-E340. doi:10.36076/ppj.2018.4.E331.

30. Yang J-. S, Chu L, Deng R, et al. Treatment of single-level thoracic tuberculosis by percutaneous endoscopic débridement and 
allograft via the transforaminal approach combined with percutaneous pedicle screw fixation: a multicenter study with a median follow-up of 36 months. World Neurosurg. 2019;122:e1472-e1481. doi:10.1016/j.wneu.2018.11.087.

31. Yu Q, Yang J, Chu L, Shi L, Deng Z, Ke Z. Clinical effectiveness of percutaneous endoscopic spinal surgery via transforaminal approach for single-level thoracic ossification of the posterior longitudinal ligament. Research Square.

32. Zeng T, Guo D. Transforaminal endoscope for the treatment of thoracic disc herniation. JCNR. 2020;4(3):9-13. doi:10.26689/ jcnr.v4i3.1205.

33. HS O, Hwang B-. W, Park S-. J, Hsieh C-. S, Lee S-. H. Percutaneous endoscopic cervical discectomy (PECD): an analysis of outcome, causes of reoperation. World Neurosurg. 2017;102:583-592. doi:10.1016/j.wneu.2017.03.056.

34. Zheng C, Huang X, Yu J, Ye X. Posterior percutaneous endoscopic cervical diskectomy: a single-center experience of 252 cases. World Neurosurg X. 2018;120:e63-e67. doi:10.1016/j. wneu.2018.07.141.

35. P-F W, Liu B-. H, Wang B, et al. Complications of fullendoscopic versus microendoscopic foraminotomy for cervical radiculopathy: a systematic review and meta-analysis. World Neurosurg. 2018;114:217-227. doi:10.1016/j.wneu.2018.03.099.

36. Ye Z-. Y, Kong W-. J, Xin Z-. J, et al. Clinical observation of posterior percutaneous full-endoscopic cervical foraminotomy as a treatment for osseous foraminal stenosis. World Neurosurg. 2017;106:945-952. doi:10.1016/j.wneu.2017.07.085.

37. PH W, Kim HS, Lee YJ, et al. Posterior endoscopic cervical foramiotomy and discectomy: clinical and radiological computer tomography evaluation on the bony effect of decompression with 2 years follow-up. Eur Spine J. 2021;30(2):534-546. doi:10.1007/ s00586-020-06637-8.

38. Youn MS, Shon MH, Seong YJ, Shin JK, Goh TS, Lee JS. Clinical and radiological outcomes of two-level endoscopic posterior cervical foraminotomy. Eur Spine J. 2017;26(9):2450-2458. doi:10.1007/s00586-017-5017-7.

39. Qiao Y, Liao W-. B, Du Q, et al. Percutaneous fullendoscopic anterior transcorporeal diskectomy for massive migrated cervical disk herniation treatment: case report and review of the literature. World Neurosurg X. 2019;132:47-52. doi:10.1016/j. wneu.2019.08.054.

40. Lin Y, Rao S, Li Y, Zhao S, Chen B. Posterior percutaneous full-endoscopic cervical laminectomy and decompression for cervical stenosis with myelopathy: a technical note. World Neurosurg X. 2019;124:350-357. doi:10.1016/j.wneu.2018.12.180.

41. Cheng X-. K, Chen B. Percutaneous endoscopic thoracic decompression for thoracic spinal stenosis under local anesthesia. World Neurosurg. 2020;139:488-494. doi:10.1016/j. wneu.2020.04.199.

42. Miao X, He D, Wu T, Cheng X. Percutaneous endoscopic spine minimally invasive technique for decompression therapy of thoracic myelopathy caused by ossification of the ligamentum flavum. World Neurosurg. 2018;114:8-12. doi:10.1016/j. wneu.2018.02.152.

43. Jia Z-. Q, X-J H, Zhao L-. T, S-Q L. Transforaminal endoscopic decompression for thoracic spinal stenosis under local anesthesia. Eur Spine J. 2018;27(Suppl 3):465-471. doi:10.1007/ s00586-018-5479-2.

Funding: The authors received no financial support for the research, authorship, and/or publication of this article.

Declaration of Conflicting Interests: The authors report no conflicts of interest in this work.

Corresponding Author: Albert E. Telfeian $\mathrm{MD}, \mathrm{PhD}$, Department of Neurosurgery, Rhode Island Hospital, Providence, RI 02903, USA; ATelfeian@ Lifespan.org

Published 27 December 2021

This manuscript is generously published free of charge by ISASS, the International Society for the Advancement of Spine Surgery. Copyright () 2021 ISASS. To see more or order reprints or permissions, see http:// ijssurgery.com. 